\title{
A complex RNA sequence detemines the internal initiation of encephalomyocarditis virus RNA translation
}

\author{
A.G.Evstafieva*, T.Y.Ugarova, B.K.Chernov ${ }^{1}$ and I.N.Shatsky \\ A.N. Belosersky Laboratory, Moscow State University, 119899, Moscow, and ${ }^{1}$ V.A. Engelhardt \\ Institute of Molecular Biology, Academy of Sciences of the USSR, 117984 Moscow, USSR
}

Received February 12, 1990; Revised and Accepted July 3, 1990

\begin{abstract}
Translation initiation on EMCV RNA occurs via binding of ribosomes to an internal sequence within the $5^{\prime}$ noncoding region. To investigate the organization of the internal ribosome entry site (IRES) we have determined the translational efficiencies of a series of deletion mutants within the $5^{\prime}$ noncoding region of EMCV RNA. Three functional regions have been distinguished: a sequence between nts $315-484$ and the upper parts of the double-helical structural domains III (nts 488-647) and IV (nts 701-763). The first one greatly enhances translation, but is not absolutely necessary for internal initiation. The other two regions are indispensable to this process. A sequence within domain IV determines inhibition of in vitro translation of mRNAs with $\mathbf{5}^{\prime}$-terminal dependent initiation. It is proposed to interact with a translational factor(s) common to the internal and 5'-terminal dependent initiation.
\end{abstract}

\section{INTRODUCTION}

For most eucaryotic mRNAs the ribosome scanning model has been proposed to explain the translation initiation process [1]. In this model 40s ribosomal subunits first bind at or near the 5 '-CAP structure and then migrate along the RNA chain until they encounter the first favourable AUG triplet, at which point translation begins. Encephalomyocarditis virus (EMCV), poliovirus and most probably all other picornavirus RNAs are translated by a different mechanism whereby ribosomes do not scan from the 5'-end of the mRNA, but bind directly to an internal sequence in the $5^{\prime}$ untranslated region (5'-UTR) and are then translocated to the initiator AUG to initiate translation $[2,3]$.

EMCV RNA is 7835 nucleotides long and the translation initiating AUG codon is located at nucleotide 834. This codon is followed by an open reading frame of 6876 nucleotides, which encodes the viral polyprotein. The initiator AUG is preceded by 10 other AUG triplets $[4,5]$.

According to the published data the 5'-terminal part of the EMCV RNA leader region up to nucleotide 338 does not affect translation; nucleotides $420-449$ do not play any essential role since the binding of cDNA fragments to the sequence between nucleotides 420 and 449 causes only slight inhibition of translation, whereas binding to sequences downstream from this position considerably inhibits translation [6]. The deletions up to nt 484 completely abolish translation in rabbit reticulocytes and HeLa cell extracts [2]. These data have been taken as an indication of the crucial role of the sequence near position 450 [6] or between nts 260 and 484 [2] for the internal initiation.

Recently a secondary structure model of the 5'-UTR of EMCV RNA has been elaborated [7]. In this model nucleotides 450-484 belong to domain III - a long double stranded helical structure containing numerous bulges (nt 450-680). From a general standpoint, the internal ribosome entry site (IRES) may contain a linear sequence signal or higher order structure(s) that are recognized by translational factors and/or ribosomal subunits. In order to clarify its nature, it is important to define more precisely if the whole domain III, part of domain III or other regions downstream are necessary for the initiation of EMCV RNA translation.

The region near nt 450 has been supposed to be the entry site for the ribosome followed by migration up to the starting AUG codon [6]. If this model is correct, one can predict that the primary structure of the downstream sequence (from IRES up to several nucleotides preceded the initiation codon) is not important for translation, and a decrease in the degree of the secondary structure of this region facilitates the scanning process. We decided to test whether this is really the case for EMCV RNA.

Another model predicts that IRES involves noncontiguous segments of RNA, resulting in the attachment of the protein factor(s) and ribosomal subunits in the immediate vicinity of the initiator AUG codon [8]. According to the secondary structure model (Fig. 3A), a sequence around nt 450 and one located not far from the initiator AUG codon find themselves in close proximity to each other. One can propose that IRES consists of the RNA segments encompassing the 'lower' parts of domains III and IV and nucleotides adjacent to them, the initiation AUG codon being rather close to this site. In order to test this possibility we decided to check if the 'upper' parts of domains III and IV are necessary for the internal initiation of translation.

In the studies described here we attempted to evaluate the functional role of specific regions in the 5'-UTR of EMC RNA and their importance for translation. Our approach was to insert cDNAs containing deletions or modifications into a T7

\footnotetext{
* To whom correspondence should be addressed
} 
transcription vector and to translate the resulting transcripts in extracts from ascites carcinoma Krebs- 2 cells. In order to reveal the specific functions of different parts of the EMCV leader region we tested the ability of its deleted forms to compete with other mRNAs in an in vitro translation system.

\section{MATERIALS AND METHODS}

Restriction enzymes and enzymes used in DNA cloning were purchased from 'Ferment' (USSR). DNA manipulations were performed by standard procedures [9]. Avian myeloblastosis virus reverse transcriptase was from Omoutninsk (USSR). T7 RNA polymerase was prepared according to [10]. pT7-2-42 containing a DNA copy of alfalfa mosaic virus (AIMV) RNA4 under control of T7 promotor was kindly provided by K.Langereis. RNAse $\mathrm{H}$ from $E$. coli was kindly provided by N.V. Chichkova, potato $X$ virus (PXV) RNA by N.A. Miroshnichenko, EMCV RNA - by A. Shabanov.

\section{Construction of plasmids}

EMCV RNA was transcribed with reverse transcriptase by a standard procedure [9] using a deoxyoligonucleotide complementary to the region $11701-1189$ as a primer. The second DNA chain was synthesized as described in[11]. Doublestranded DNA was completely digested by Msp1 and the fragment corresponding to nucleotides $315-1155$ was cloned in the Acc1 site of pTZ18R (Pharmacia). Two clones, pTE1 and pTE2, containing the inserted fragment in the direct and reverse orientations respectively, were isolated.

Plasmid pTE3 was derived from pTE1 by deletion of the EcoRl-Xbal polylinker sequence. EcoRl and Xbal sites were made blunt-ended with nuclease $\mathrm{S} 1$ before religation.

pTE4 was constructed by insertion of pTE2 EcoRl-HindIII fragment, representing bases $485-1155$ of the viral genome, that was made blunt-ended with T4 DNA polymerase, ligated with EcoRl-linkers and digested by EcoRl+BamHl, into EcoR1-BamH1 sites of pTZ18R.

To obtain pTE5, plasmid pTE2, first completely digested by Bgl1, and then made blunt-ended with T4 DNA polymerase, ligated with EcoRl linkers and digested by EcoRl+BamHl, was fractionated in $1 \%$ agarose gel and a 592 b.p. fragment was inserted into EcoRl-BamHl sites of pTZ18R.

To obtain pTE6, the smaller HindIII-HindIII fragment of pTE1 was digested with Hinfl, filled in with the Klenow fragment of DNA polymerase 1, ligated with EcoRl linkers, digested by EcoRl+Pstl and inserted into EcoRl-Pstl sites of pTZ18R.

To construct pTE7, pTE3 was partially digested with HindIII, the linear form was filled in with the Klenow fragment and ligated with EcoRl linkers. Colonies were screened by the appearance of only one EcoRl site at the distance 670 nts from the Pstl site.

pTE8 was constructed by inserting the pTE6 EcoRl-Pstl smaller fragment, representing bases $647-1155$ of the viral genome, into EcoRl-Pstl sites of pTE7.

pTE9 was derived from pTE3 by deletion of Kpnl-Kpnl fragment ( 250 b.p.), which was replaced with the EcoRl linker.

To obtain pTE10, the smaller HindIII-HindIII fragment of pTEl was partially digested by Taql, the 392 b.p. fragment corresponding to sequence $763-1155$ was filled in with the Klenow fragment, ligated with EcoRl linkers, digested by EcoRl+Pstl and cloned into EcoRl-Pstl sites of pTE9.

pTE11 was constructed by ligation of the isolated Sau3ASau3A fragment of pTEl, corresponding to nucleotides 724-907, into BamHl site of pTZ18R. Screening was based on the appearance of 250 b.p. product after Kpnl digestion.

pTEl2 was derived from pTE3 by deletion of Kpnl-Kpnl fragment ( 250 b.p.).

pTEl3 was constructed by ligation of the Kpnl-Kpnl fragment from pTE11 (250 b.p.) into the Kpnl site of pTEl2. The orientation of the inserted fragment was checked by the appearance of a 450 b.p. Smal-Pstl digestion product.

pTEl4 was constructed by ligation of EcoRl-Pstl fragment of pTEl0, corresponding to sequence $763-1155$ of the viral genome, into EcoRl-Pstl sites of pTZ18R.

\section{T7 polymerase in vitro transcription}

Transcription was carried out in a reaction mixture $(100 \mu \mathrm{l})$ containing $5 \mu \mathrm{g}$ of a plasmid, linearized by a suitable restriction enzyme, $40 \mathrm{mM}$ Tris- $\mathrm{HCl}$ (pH 7.5), $6 \mathrm{mM} \mathrm{MgCl}, 2 \mathrm{mM}$ spermidine, $10 \mathrm{mM} \mathrm{NaCl}, 10 \mathrm{mM}$ DTT, $1 \mathrm{mM}$ of each rNTP, and $20-50 \mathrm{U}$ of $\mathrm{T} 7 \mathrm{RNA}$ polymerase at $37^{\circ} \mathrm{C}$ for $45 \mathrm{~min}$. The reaction was stopped by phenol-chloroform extraction ( 3 times) and ethanol precipitation ( 2 times). The transcripts obtained were analyzed in denaturing $4 \%$ PAAG.

\section{Probing the structure of transcripts}

Partial digestion by ribonuclease was performed in reaction mixtures $(20 \mu \mathrm{l})$ containing: $5 \mu \mathrm{g}$ of RNA, $5 \mathrm{mM} \mathrm{MgCl}, 10$

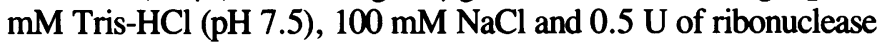
T2 (Sigma); or $5 \mu \mathrm{g}$ of RNA, $10 \mathrm{mM} \mathrm{MgCl}_{2}, 20 \mathrm{mM}$ Tris- $\mathrm{HCl}$ (pH 7.5), $200 \mathrm{mM} \mathrm{NaCl}$ and $20 \mathrm{ng}$ of ribonuclease from cobra venom (Minor Bio, USSR). After incubation for $30 \mathrm{~min}$. at $20^{\circ} \mathrm{C}$, the mixture was extracted with phenol-chloroform ( 3 times) and ethanol precipitated ( 2 times).

The sites of ribonuclease hydrolysis and dideoxysequencing of transcripts were determined by reverse transcription [12, 13], using synthetic oligodeoxynucleotides complementary to nucleotides $826-842,653-669,460-477$ and $712-744$ as primers.

The FOLD program [14, see also 15] was used to construct the secondary structure models.

\section{In vitro translation}

Synthetic RNAs were translated in a reaction mixture $(25 \mu \mathrm{l})$ containing $18 \mathrm{mM}$ Tris- $\mathrm{HCl}(\mathrm{pH} 7.6), 51 \mathrm{mM} \mathrm{KCl}, 120 \mathrm{mM}$ $\mathrm{K}$-acetate, $2.1 \mathrm{mM} \mathrm{Mg}$-acetate, $0.1 \mathrm{mM}$ spermidine, $1.5 \mathrm{mM}$ ATP, $0.1 \mathrm{mM}$ GTP, $10 \mathrm{mM}$ creatine phosphate, $80 \mu \mathrm{g} / \mathrm{ml}$ creatine kinase, $40 \mu \mathrm{M}$ each of unlabeled amino acids, $20 \mu \mathrm{Ci}$ $\left[{ }^{35} \mathrm{~S}\right]$ methionine, $0.3-1.0 \mu \mathrm{g}$ of RNA and $15 \mu \mathrm{l}$ of S30 extract from ascites carcinoma Krebs 2 cells [16], gel-filtered through a Sephadex G-25 column. The reaction mixtures were incubated at $30^{\circ} \mathrm{C}$ for $60 \mathrm{~min}$. Products of in vito translation were analyzed on gradient SDS-PAA gels (8-20\%), the gels were fixed in $10 \%$ ethanol-7.5\% acetic acid, dried, exposed to X-ray film and quantified by soft laser densitometry (LKB).

\section{RESULTS}

\section{Constructions}

To obtain the region of the 5'-UTR of EMCV RNA significant for cap-independent translation we inserted the EMCV cDNA fragment comprising nucleotides 315-1155 into a T7-transcription vector since the sequence encompassing nucleotides $1-338$ was shown to be dispensable to translation [6]. The fragment 315-1155 contains a noncoding region 
comprising nucleotides $315-833$, a polyprotein start site and a coding region 322 nucleotides long. Then we made several deletions in the 5 '-noncoding region (Fig 1).

Each plasmid was linearized by treatment with a suitable restriction enzyme (as shown in Fig. 1) and the runoff transcripts were synthesized by $\mathrm{T} 7$ polymerase. Each transcript was analyzed on 4\% PAA gels containing 7M urea and shown to be intact and of the expected size (data not shown). The primary structure of the 5'-noncoding region of the basic transcript $(\operatorname{tr} 315)$ and the deletion sites in the mutant transcripts were confirmed by RNA sequencing.

Tr485, tr563, tr647 and tr763 have the same coding regions as $\operatorname{tr} 315$, but their leader regions are consequently shortened from the 5'-end (Fig.1). All the tr $\Delta$ have internal deletions in the 5 '-UTR, the decanucleotide GGGAAUUCCC being inserted between U-488 and A-647 into tr $\Delta 488-647$, and between G-701 and C-763 into tr $\Delta 701-763$; and the trinucleotide GGG being inserted between C-707 and G-723 into tr $\Delta 707-723$. All the transcripts have short nonviral sequences at both $5^{\prime}$ and $3^{\prime}$ ends, originating from the vector.

\section{Secondary structure}

We probed the stem and loop structure of the following segments of the tr315 5'-noncoding region: $347-450,510-620,620-680$ and 680-800 with two enzymes: RNase T2, specific to the RNA single-stranded sites, and RNase VI from cobra venom which cleaves the RNA backbone in a helical conformation [17, see also 15]. The cleaved bases were detected by primer extention using four different oligonucleotides as primers. The results of our probing experiments (shown in Fig. 3A) are in agreement with the secondary structure model of the conservative part of EMCV RNA 5'UTR, elaborated by E.Pilipenko et al. [7]. We therefore conclude that the secondary structure of $\operatorname{tr} 315$ 5 '-noncoding leader is the same, at least in general characteristics, as in the native EMCV RNA.

When we analyze the function of transcripts with deletions, the question arises whether a deletion causes structural transition only in the particular domain, part of which has been deleted, or it induces a change in the stem and loop stucture of other domains. In order to answer this question we probed the structure

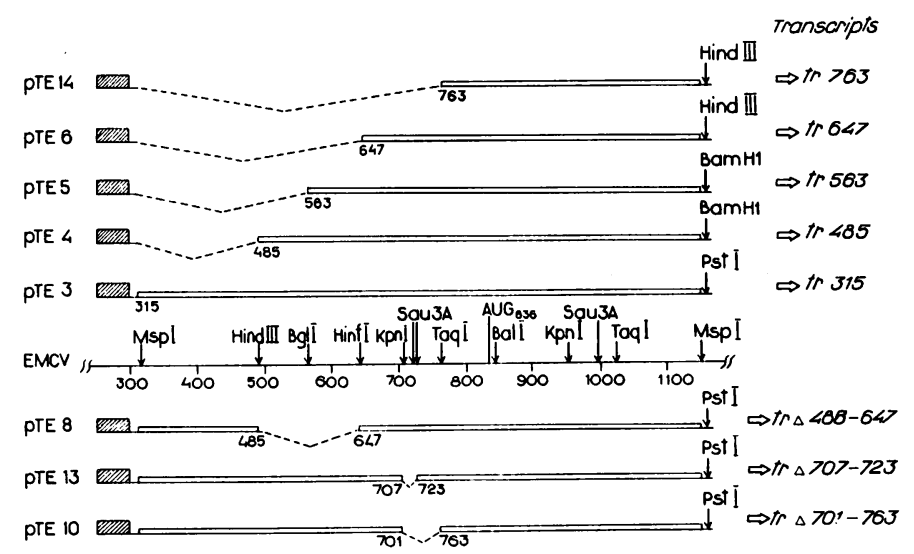

Figure 1. Schematic representation of plasmids with deletions in the 5 -UTR of EMCV RNA and corresponding transcripts. The hatched box represents T7 promoter; the blank box is EMCV sequence. The numbers below the constructs represent distances in nucleotides. To obtain tr315-485 and tr315-837, the plasmid pTE3 was linearized by HindIII and Bal1, respectively. of the leader regions of $\operatorname{tr} 485, \operatorname{tr} \Delta 488-647, \operatorname{tr} \Delta 701-763$ and $\operatorname{tr} \Delta 707-723$ using RNases T2 and V1. The results obtained, examples of which are shown in Fig. 2, are summarized in Fig. $3 \mathrm{~A}-\mathrm{E}$. The sites sensitive to RNase $\mathrm{T} 2$ and $\mathrm{V} 1$ in the domains I and II proved to be the same for $\operatorname{tr} \Delta 701-763, \operatorname{tr} \Delta 707-723$, $\operatorname{tr} \Delta 488-647$ and tr315 (Fig. 2); in the domain III - the same for $\operatorname{tr} \Delta 701-763, \operatorname{tr} \Delta 707-723$ and $\operatorname{tr} 315$; in the domain IV RNases T2 and V1 cut tr 485 and tr $\Delta 488-647$ at the same sites as $\operatorname{tr} 315$ (as shown in Fig. 3A). Therefore in all the four cases we did not detect any changes in the secondary structure of the domains not affected by deletions.

Then we computer-analyzed the stem and loop structure of domains with deletions, i.e. the domain III of tr485 and $\operatorname{tr} \Delta 488-647$, and the domain IV of $\operatorname{tr} \Delta 701-763$ and tr $\Delta 707-723$. A comparison of the results obtained with the enzymatic probing data enabled us to construct secondary structure models for these domains (Fig. 3 B, C, D, E).

\section{Translation}

Transcripts were translated in vitro in extracts from ascites carcinoma Krebs- 2 cells. The translational products were resolved by SDS-PAAG electrophoresis. Care was taken in each case to ensure that apparent differences in translational efficiency were not the result of variable RNA concentrations.

Translation of tr315 was very efficient, and tr315 was able to compete with EMCV RNA in a cell-free system [Fig. 5].

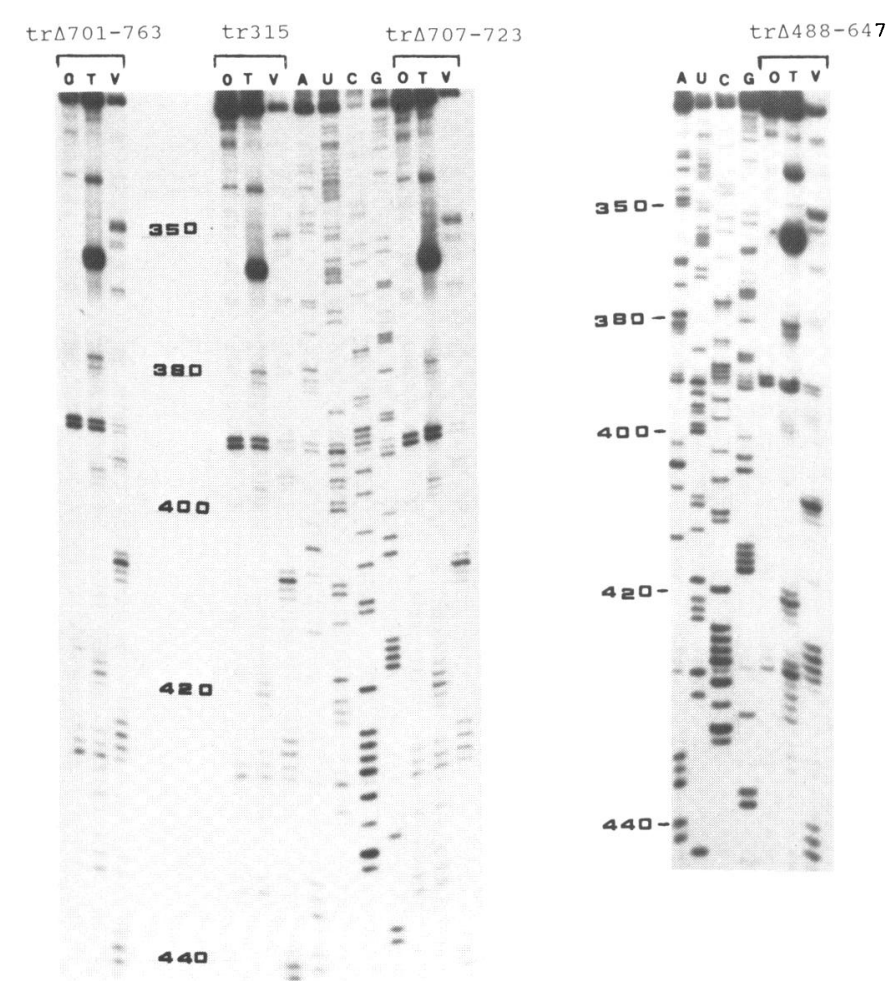

Figure 2. An example of probing of the secondary structure of the region comprising nucleotides $315-440$ in $\operatorname{tr} \Delta 701-763, \operatorname{tr} 315, \operatorname{tr} \Delta 707-723$ and $\operatorname{tr} \Delta 488-647$. Transcripts were treated with ribonuclease T2 (lanes T) and V1 (lanes V) as descibed under Materials and Methods and then they were used as templates for the oligonucleotide-primed cDNA synthesis by reverse transcriptase. Lane $O$ corresponds to the nontreated RNA samples. Lanes A, U, G and C contains the products of the corresponding dideoxy sequencing reactions. Numbering to the side of the gel refers to sequence position on the EMCV RNA. 

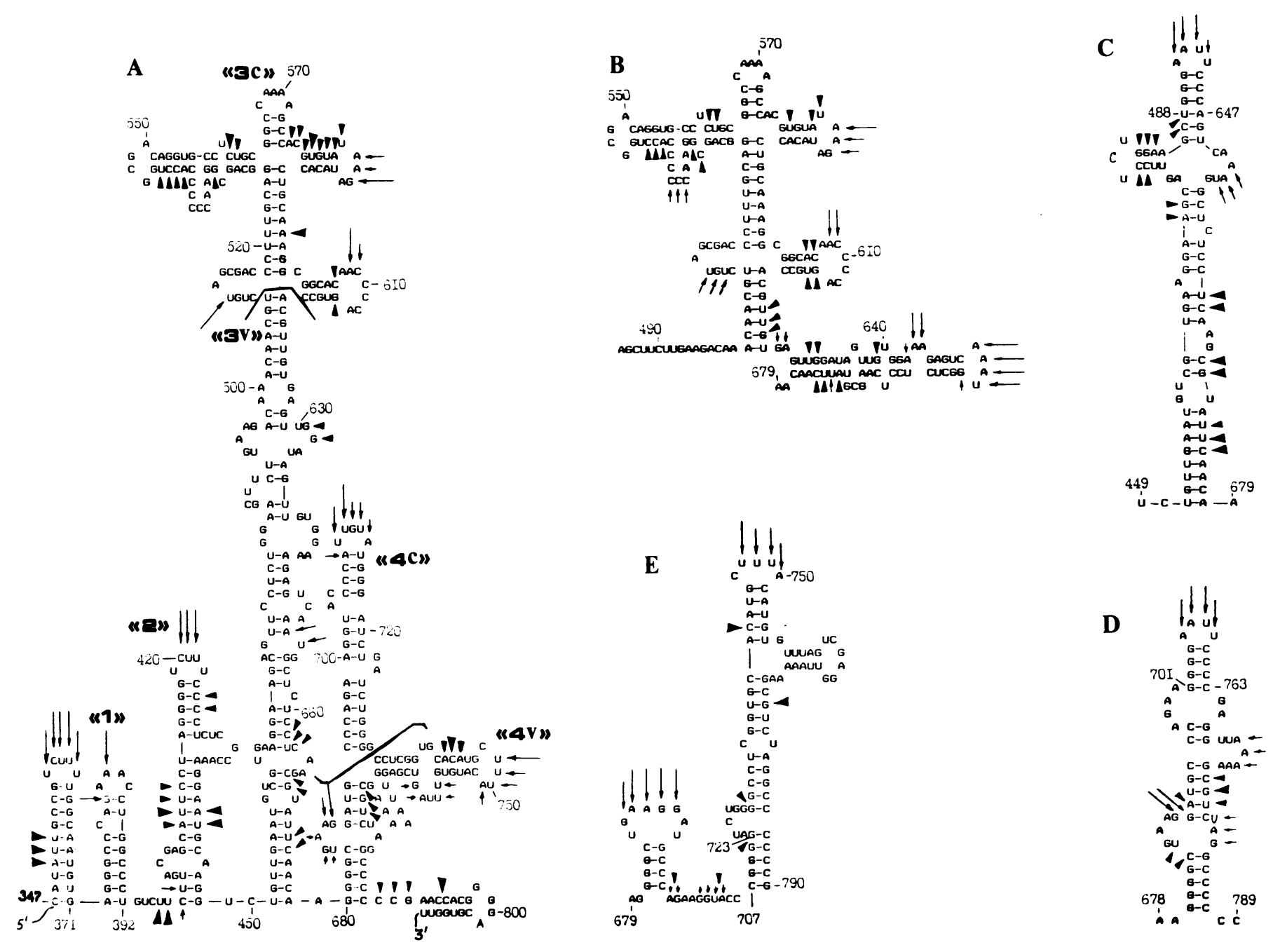

Figure 3. The secondary structure models of:

A-the conservative part of EMCV RNA 5'-UTR, elaborated by E. Pilipenko et al. [7], combined with the results of probing of the tr 315 secondary structure; B-the domain III of tr485;

C-the domain III of $\operatorname{tr} \Delta 488-647$;

D-the domain IV of $\operatorname{tr} \Delta 701-763$;

E-the domain IV of $\operatorname{tr} \Delta 707-723$;

arrows show the sites cleaved by ribonuclease T2 $(\rightarrow)$ and V1 (4)

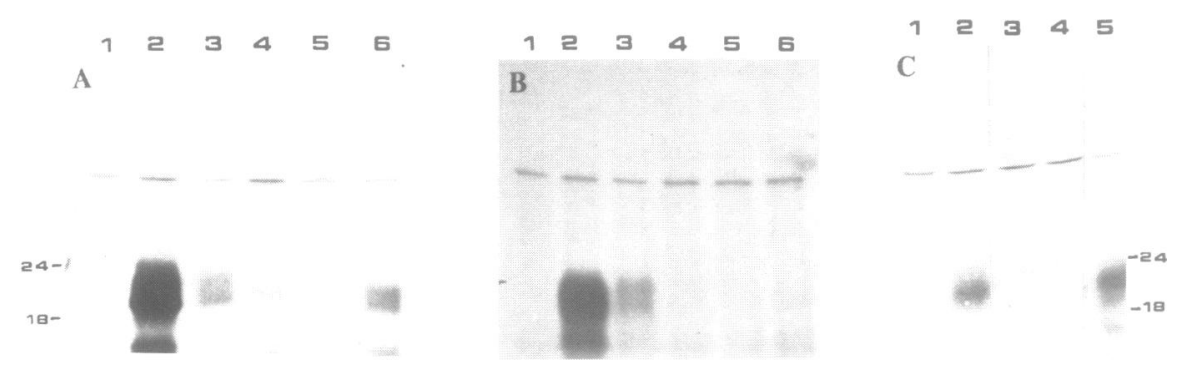

Figure 4. Translation in the extracts from Krebs 2 cells of:

A: $1-\mathrm{H}_{2} \mathrm{O} ; 2-\operatorname{tr} 315 ; 3-\operatorname{tr} 485 ; 4-\operatorname{tr} 563 ; 5-\operatorname{tr} 647 ; 6-\operatorname{tr} 763 ; \mathrm{B}: 1-\mathrm{H}_{2} \mathrm{O} ; 2-\operatorname{tr} 315 ; 3-\operatorname{tr} 485 ; 4-\operatorname{tr} \Delta 701-763 ; 5-\operatorname{tr} \Delta 707-723 ; 6-\operatorname{tr} \Delta 488-647 ; \mathrm{C}: 1-\mathrm{H}_{2} \mathrm{O}$ $2-\operatorname{tr} 315 ; 3-\operatorname{tr} 484 ; 4-\operatorname{tr} 563 ; 5-\operatorname{tr} 763$. Translations were performed as described in 'Materials and Methods', except that $120 \mathrm{mM}$ KAc was ommited from the reaction mixture in $\mathrm{C}$ and the gels were impregnated in $1 \mathrm{M}$ sodium salicylate in $\mathrm{A}$ and $\mathrm{B}$. Molecular mass markers are shown in kilodaltons.

Therefore it contains all the necessary signals for the initiation process. The product of $\operatorname{tr} 315$ translation proved to be rather heterogeneous and exhibited an unexpectedly low electrophoretic mobility (m.w. 18-24 kD, Fig. 4, lane 2). Translation of $\operatorname{tr} 315$ was predicted to produce a single polypeptide with a molecular weight of $12-13 \mathrm{kD}$ containing the EMCV leader polypeptide (m.w. $7900 \mathrm{D})$ fused to the truncated capsid protein $\sigma$.

Computer-assisted analysis showed the absence of open reading 
frames in tr315 coding for polypeptides of higher molecular weight. The predominant translational product of EMCV RNA, cleaved with $\mathrm{RNase} \mathrm{H}$ in the polyprotein coding region between positions 1170 and 1189 (i.e. approximately near the point of $\operatorname{tr} 3153^{\prime}$-end), was found to be qualitatively identical with that of tr315 translation (not shown). Tr315-837 truncated just after the native initiation codon displayed no translational activity.

We conclude that the truncated 5'-noncoding leader in tr315 directs the initiation of translation from the same start site as the intact 5'-leader region of EMCV RNA and that here we are actually dealing with the anomalous electrophoretic mobility of the tr315 translational product. The possibility of polypeptide chain modification in an in vitro system resulting in a change of its electrophoretic mobility is confirmed by an anomalous behaviour in SDS-PAA gels of the EMCV leader polypeptide with a predicted molecular weight of $7.9 \mathrm{kD}$ earlier detected as two bands of 12 and $14 \mathrm{kD}$ [18]. So in this work we estimated the efficiency of translation of different mutants in the EMCV $5^{\prime}$-UTR by synthesising the product described above. The heterogeneity of this product can be explained by the absence of the terminator codon in $\operatorname{tr} 315$ so that the ribosomes carrying peptidyl-tRNAs with progressively shorter polypeptides accumulate in its coding region.

Translation of tr485 (truncated up to nt485) was about $1 / 10$ as efficient as that of $\operatorname{tr} 315$ (Fig. $4 \mathrm{~A}, \mathrm{~B}$, lane 3 ). The removal from the $\operatorname{tr} 3155^{\prime}$-end of nucleotides $315-562$ in tr563 and nucleotides 315-646 in tr647 made their translation undetectable (Fig. 4A, lanes 4, 5). The removal of the most part of EMCV 5 -noncoding sequences (nucleotides $315-762$ ) in tr763 restored RNA translatability, but with very low efficiency (Fig. 4A, lane 6).

Therefore the successive shortening of EMCV 5'-UTR results in decrease, abolition and restoration, albeit at low efficiency of translational activity of the truncated mRNAs. This may be reasonably explained by a switch over from the internal to the 5 -end dependent initiation of translation as was shown for deletion mutants in the $5^{\prime}$-UTR of poliovirus [19]. When the length of the EMCV 5'-noncoding leader is intermediate as in $\operatorname{tr} 563$ and tr647, it makes both types of initiation impossible in an in vitro system.

In order to weaken the secondary structure of transcripts we decreased the ionic strength in extracts from $170 \mathrm{mM} \mathrm{K} \mathrm{K}^{+}$, optimal for EMCV RNA translation of uncapped cellular mRNAs [20]. As expected, this resulted in an increase in the efficiency of tr763 translation, and in a decrease in the efficiency of $\operatorname{tr} 315$ and tr485 translation, in the case of tr485 to the undetectable level; tr563 remained 'silent' (Fig. 4 C).

Deletion of the internal sequences comprising nucleotides $702-762,708-722$ and $489-646$ produced $\operatorname{tr} \Delta 701-763$, $\operatorname{tr} \Delta 707-723$ and $\operatorname{tr} \Delta 488-647$, respectively. They failed to be translated under conditions optimal for EMCV RNA translation (Fig. 4 B, lanes 4, 5, 6). This result shows that initiation of $\operatorname{tr} 315$ translation can hardly be explained in terms of the scanning model.

\section{Competition experiments}

In order to distinguish somehow the specific functions of different parts of the EMCV 5'-UTR, we investigated the competition of the deletion variants of $\operatorname{tr} 315$ with three other mRNAs: the intact EMCV RNA, potato X virus (PXV) RNA (cap-specific initiation) and noncapped alfalfa mosaic virus (AlMV) RNA 4 (5'-terminal dependent initiation). $\operatorname{Tr} 315$ inhibited significantly the translation of all of them (Figs. 5, 6, 7). The inhibition observed was not

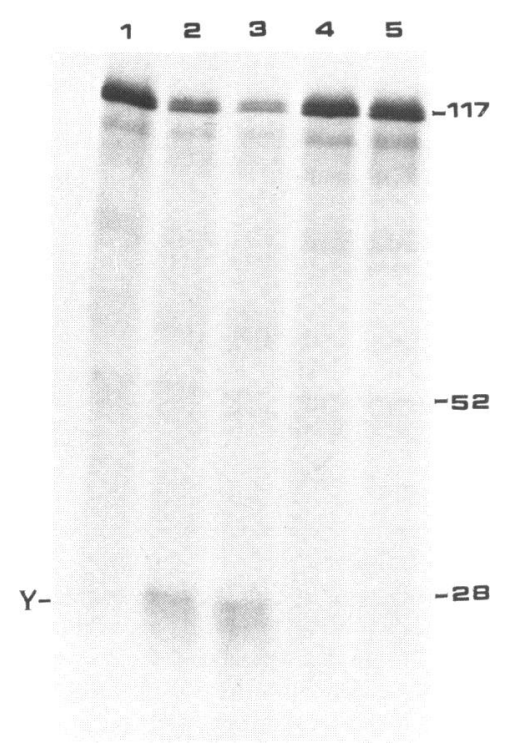

Figure 5. Translation of EMCV RNA $(2 \mu \mathrm{g})$ in the presence of: $1-\mathrm{H}_{2} \mathrm{O} ; 2$, 3-tr315 (0.25 and $0.5 \mu \mathrm{g}) ; 4,5-\operatorname{tr} 484(0.25$ and $0.5 \mu \mathrm{g})$. Molecular mass markers are shown in kilodaltons. The position of $\operatorname{tr} 315$ translation product $(\mathrm{y})$ is shown.

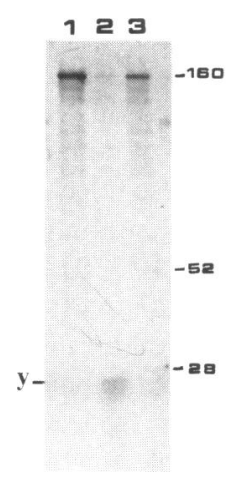

Figure 6. Translation of PXV RNA $(1.7 \mu \mathrm{g})$ in the presence of: $1-\mathrm{H}_{2} \mathrm{O}$; $2-\operatorname{tr} 315(0.5 \mu \mathrm{g})$; 3- $\operatorname{tr}$ anti315 $(0.5 \mu \mathrm{g})$. Molecular mass markers are shown in kilodaltons. The position of $\operatorname{tr} 315$ translation product $(\mathrm{y})$ is shown).

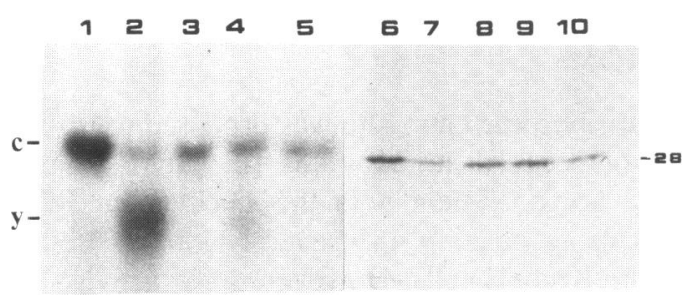

Figure 7. Translation of AlMV RNA4 $(1.0 \mu \mathrm{g})$ in the presence of: $1,6-\mathrm{H}_{2} \mathrm{O}$; $2-\operatorname{tr} 315 ; \quad 3, \quad 10-\operatorname{tr} 315-837 ; \quad 4-\operatorname{tr} 485 ; \quad 5-\operatorname{tr} 563 ; \quad 7-\operatorname{tr} \Delta 488-647$; $8-\operatorname{tr} \Delta 701-763 ; 9-\operatorname{tr} \Delta 707-723(0.5 \mu \mathrm{g}$ of each transcript). The positions of migration of the AlMV coat protein (C) and tr315 translation product (Y) are shown.

connected with unspecific overloading of the translation system with polynucleotdies. Indeed, tr315 inhibited translation of EMCV RNA, but the same concentration of tr484 did not (Fig. 5). Translation of PXV RNA was inhibited by tr315, but it was not inhibited by the same amount of a nonspecific untranslatable 
Table 1. Competition of different transcripts with mRNAs in the in vitro translation

\begin{tabular}{|c|c|c|c|c|c|c|}
\hline \multirow[t]{2}{*}{ Transcript } & \multicolumn{3}{|c|}{ Domain } & \multicolumn{3}{|c|}{$\begin{array}{c}\text { Inhibition of translation of } \\
\text { RNAs }\end{array}$} \\
\hline & I,II & III & IV & PXV & AlMV & EMCV \\
\hline $\operatorname{Tr} 315$ & $\mathrm{i}$ & $\mathrm{i}$ & $\mathrm{i}$ & + & + & + \\
\hline $\operatorname{Tr} 485$ & $\mathrm{~d}$ & d & i & + & + & - \\
\hline $\operatorname{Tr} 563$ & $\mathrm{~d}$ & d & $\mathrm{i}$ & + & + & - \\
\hline Tr647 & $\mathrm{d}$ & d & $\mathrm{i}$ & + & + & - \\
\hline $\operatorname{Tr} 763$ & $\mathrm{~d}$ & $\mathrm{~d}$ & d & - & - & - \\
\hline $\operatorname{Tr} \Delta 488-647$ & $\mathrm{i}$ & d & $\mathrm{i}$ & + & + & NT \\
\hline $\operatorname{Tr} \Delta 701-763$ & $\mathrm{i}$ & $\mathrm{i}$ & d & - & - & NT \\
\hline $\operatorname{Tr} \Delta 707-723$ & $\mathrm{i}$ & $\mathrm{i}$ & d & - & - & NT \\
\hline $\operatorname{Tr} 315-484$ & $\mathrm{i}$ & $\mathrm{d}$ & d & - & - & - \\
\hline $\operatorname{Tr} 315-837$ & $\mathrm{i}$ & $\mathrm{i}$ & $\mathrm{i}$ & + & + & + \\
\hline
\end{tabular}

(i-intact, d-deleted or destroyed; NT-not tested)

Radioactive bands corresponding to EMCV, PXV and AlMV protein products (see figs. 5, 6, 7 and not shown) were quantified by soft-laser densitometry (LKB). The symbols ' + ' or ' - ' mean that the ratio of the corresponding bands in the presence and absence of the added transcript is in the range $0.2-0.5$ or $0.8-1.2$, respectively. Translations for each experiment were performed at least twice with different RNA preparations.

tr anti315 (Fig. 6). We observed a nonspecific inhibition of translation only when the total concentration of polynucleotides added to the reaction mixture was more than $100 \mu \mathrm{g} / \mathrm{ml}$. We never exceeded this value in subsequent experiments.

The results of the competition experiments (Figs. 5, 6, 7 and not shown) are summarized in Table 1. From it one may conclude that the ability of the truncated of PXV RNA and A1MV RNA 4 correlates with the integrity of domain IV. Indeed, tr $484, \operatorname{tr} 563$ and tr647 do compete with these mRNAs, but tr763, $\operatorname{tr} \Delta 701-763$ and $\operatorname{tr} \Delta 707-723$ do not. Our probing experiments demonstrate that both deletions in domain IV (701-763 and 707-723) cause a structural change only in this particular domain, but they do not alter the structure of the entire initiator segment. Therefore either the intact structure of domain IV or some nucleotide sequence located downstream from nt 647 and overlapping the region 708-722 is responsible for the inhibition of PXV RNA and AIMV RNA 4 translation.

On the other hand, the region between nts 315 and 837 is necessary for the inhibition of EMCV RNA translation. None of the fragments $315-484$ and $484-837$ has this property.

\section{DISCUSSION}

The internal ribosome entry site was proposed to reside around nt 450 [6] or between nts 260-484 [2]. At first glance some of our data are consistent with this conclusion. Removal of nucleotides up to position 485 severely inhibits translation in extracts from Krebs 2 cells. However, some activity persists in tr485 and it is fully provided for by internal initiation: under conditions optimal for the 5 -terminal dependent initiation, the activity of tr 485 proved to be undetectable. Therefore we sought for other sites within 5'-UTR which might be of significance for internal initiation.

We showed that deletion of nucleotides $315-484$ in tr485 results in a serious change of the conformation of the domain III 'lower' part (nucleotides 626-678, Fig. 3B), the structure of the 'upper' $(3 \mathrm{C})$ part being the same as in tr315. Its translational efficiency may decline as a result of the disappearance of some nucleotide sequence or/and disruption of some higher order structure important for translation. We cannot choose one definite option, probably both take place. When we deleted 78 additional nucleotides that should have disrupted the structure of both ' $3 \mathrm{C}$ ' and ' $3 \mathrm{~V}$ ' parts of domain III in tr563, it completely failed to be translated. When only the 'upper' part of domain III was deleted (tr $\Delta 488-647)$ without serious changes in the structure of its 'lower' part (Fig. 3C), the synthesis of the characteristic heterogeneous translation product also proved to be undetectable.

We conclude that the 'upper' part of domain III is necessary for EMCV-specific initiation of translation. The structural intactness of its 'lower' part may enhance translation but is not absolutely necessary for it.

In order to test the function of the domain IV, two other deletion mutants have been constructed. In the first one, $\operatorname{tr} \Delta 701-763$, a conservative hairpin ' $4 C$ ' and a hairpin $739-760$ from the region ' $4 \mathrm{~V}$ ' were deleted, the structure of the domain IV 'lower' part being the same as in tr315 (Fig. 3D). The second one, $\operatorname{tr} \Delta 707-723$, had a rather small deletion 15 nucleotides long; instead, trinucleotide GGG was inserted, which resulted in the disappearance of the ' $4 \mathrm{C}$ ' hairpin and in significant structural changes in the 'lower' part of domain IV, the hairpin with nucleotides $741-755$ being the same as in tr 315 (Fig. 3E). Despite the intactness of all other domains in these two mutants, both of them failed to be translated.

This result allowed us to reject the simplified mechanism of internal initiation of EMCV RNA translation whereby 40s subunits first bind at IRES inside the 5'-UTR located in or upstream of domain III (according to [6] and [2]) and then migrate along the RNA chain until they encounter the first favourable AUG codon, at which point translation begins. In this case one could predict that: 1)the primary structure of a sequence comprising nucleotides 680-790 (domain IV) must not be important for translation; 2 ) a decrease in the number of base pairs in the domain IV in $\operatorname{tr} \Delta 707-723$ and $\operatorname{tr} \Delta 701-763$ compared with that in tr315 (free energies -48.1, - 34.1 and $-53.2 \mathrm{kcal}$, respectively) must facilitate the scanning process. Instead, both deletion mutants failed to be translated. This means that the mechanism of internal initiation of EMCV RNA translation is more complicated than it was thought to be before, and the extensive region containing both domains III and IV is necessary for this process.

Therefore the first model for the internal initiation of translation discussed by S.K. Jang et al. [8] is rather unlikely, at least concerning the scanning of RNA by the ribosomal subunit from the IRES element up to the initiation AUG codon according to the rules of $5^{\prime}$-end dependent translation. Alternatively, the second model predicts that IRES involves noncontiguous segments of RNA, resulting in the attachment of the protein factor and the ribosome in the immediate vicinity of the initiatior AUG codon. According to the secondary structure model discussed above (Fig. 3A), a sequence around nt 450 , considered to be crucial for the internal initiation of translation [6], and one located not far from the initiator AUG codon, find themselves in close proximity to each other. One could think that IRES consists of the RNA segments encompassing the 'lower' parts of domains III and IV and nucleotides adjacent to them, the initiation AUG codon being rather close to this site. However, such a model cannot explain the lack of translational activity of RNAs in which the 'upper' part of either domain III or domain IV is deleted without significant structural changes in the 'lower' part. That is why we suspect that IRES segments may be connected not only by the secondary structure itself, but also by tertiary interactions. 
Finally, we can suggest one more explanation for such a complex structure of IRES, i.e., that the internal initiation of translation is a multistep process which needs interaction of more than one factor with the initiator region and maybe long-range conformational transition(s) in the RNA, induced by factor(s). Our competition experiments seem to favour such a suggestion. A sequence with domain IV was shown to be responsible for the inhibition of the in vitro translation of PXV RNA and AlMV RNA4, possessing 5'-terminal dependent initiation. On the other hand, this sequence is not sufficient for the inhibition of EMCV RNA translation, a region $315-837$ being necessary for that.

We explain such inhibition by a competition for some translational components and speculate that domain IV interacts with a translation factor that is common to both the 5 '-end dependent (PXV RNA and AlMV RNA4) and the internal (EMCV RNA) initiation of translation. Interaction with this factor is probably necessary for the initiation of EMCV RNA translation-this might explain the fact that $\operatorname{tr} \Delta 701-763$ and $\operatorname{tr} \Delta 707-723$ failed to be translated. But this factor is not limiting for EMCV RNA translation as a number of transcripts with the intact domain IV (tr485, tr563, tr647) cannot compete with EMCV RNA.

We expect that another translation factor (component) interacts with EMCV RNA in the region between nucleotides 315 and 837 .

The initiation segment of poliovirus RNA has some properties similar to that of EMCV. Trono et al. [21] have localized an extensive region between nucleotides 320 and 631 (region P) as a major determinant to mediate cap-independent translation. The fact that small alterations in the region $P$ resulted in the abolition or modification of its function has led to the suggestion that region $P$ forms a large coherent structure [22]. Some evidence has been presented that this functional unit cannot be considered in a linear fashion; it is probably the secondary structure that has a role to play. And finally, cell proteins have been shown to bind to at least two sites within the 5'-UTR of poliovirus RNA [23].

Therefore, the great length, the complex secondary structure comprising several domains and the existence of more than one binding sites for translational components may be the common features of the internal ribosome entry site.

In conclusion, within EMCV RNA 5'-UTR we may single out at least three main functional parts: the sequence between $\mathrm{nt} 315$ and 485, the upper part of domain III and domain IV. The first one greatly enhances translation of EMCV RNA, but is not absolutely necessary for internal initiation. The other two are indispensable to this process. These parts may be recognized by different translational components. Actually we have detected in the extracts from Krebs- 2 cells a protein that specifically binds to the 5'-UTR of EMCV RNA between nts 315 and 484 (to be published elsewhere). Investigation of its function and a search for other factors will be the main objective of further studies.

When this paper was in preparation M.T. Howell et al. reported the direct evidence that the correct initiation site, the 11-th AUG from the EMCV RNA 5'-end, is not selected by a mechanism involving scanning from an upstream entry site [24]. This is completely in agreement with our conclusion about the complex structure of the EMCV RNA internal ribosome entry site comprising several hundred nucleotides.

\section{ACKNOWLEDGEMENTS}

We are indebted to Prof. A.A. Bogdanov for his encouragment and support, to $\mathrm{N}$. Sonenberg for the critical reading of the manuscript and useful discussions. We thank T. Bystrova for excellent technical assistance, A. Popravko for help in DNA cloning and Dr. A. Bakin for help in preparation of the manuscript.

\section{REFERENCES}

1. Kozak,M. (1989) J. Cell. Biol. 108 229-241.

2. Jang,S.K., Krausslich,H.G., Nicklin,M.J.H., Duke,G.M., Palmenberg,A.C. and Wimmer,E. (1988) J. Virol. 62, 2636-2643.

3. Peletier,J. and Sonenberg,N. (1988) Nature 334, 320-325.

4. Palmenberg,A.C. (1987) UCLA Symp. Mol. Cell. Biol. 54, 25-34.

5. Palmenberg,A.C., Kirby,E.M., Janda,M.R., Drake,N.L., Duke,G.M., Portratz,K.F. and Colett,M.S. (1984) Nucleic Acids Res. 12, 2969-2985.

6. Shih,D.S., Park,I.W., Evans,C.L., Jaynes,J.M. and Palmenberg,A.C. (1987) J. Virol. 61, 2033-2037.

7. Pilipenko,E.V., Blinov,V.M., Chernov,B.K., Dmitrieva,T.M. and Agol,V.I. (1989) Nucl. Acids Res. 17, 5701-5711.

8. Jang,S.K., Davies,M.V., Kaufman,R.J. and Wimmer,E. (1989) J. Virol. 63, $1651-1660$.

9. Maniatiis,T., Fritch,E.F. and Sambrook,J. (1982) Molecular Cloning: $a$ laboratory manual. Cold Spring Harbor Laboratory, Cold Spring Harbor, N.Y.

10. Tabor,S. and Richardson,C.C. (1975) Proc. Natl. Acad. Sci. USA 82, 1074-1078.

11. In: DNA Cloning. Ed. by Glover,D.M., IRL Press, Oxford, Washington DC., v.1, 79-84.

12. Barta,A., Steiner,G., Brosius,J., Noller,N.F. and Kuechller,E. (1984) Proc. Natl. Acad. Sci. USA 81, 3607-3611.

13. Shelness,G.S. and Williams,D.L. (1985) J. Biol. Chem. 260, 8637-8646.

14. Zuker,M. and Steigler,P. (1981) Nucl. Acids Res. 9, 133-148.

15. Skinner,M.A., Racaniello,V.R., Dunn,G., Cooper,Ju., Minor,P.D. and Almond,J. (1989) J. Mol. Biol. 207, 379-392.

16. Karasev,A.V., Miroshnichenko,N.A. and Ugarova,T.Yu. (1989) Molek. Biol. (russ) 23, 119-126.

17. Ehresmann,C.E., Baudin,F., Mougel,M., Romby,P., Ebel,J.P. and Ehresmann,B. (1987) Nucleic Acids Res. 15, 9109-9127.

18. Kazachkov,Ju.S., Chernovskaya,T.V., Syanova,E.Yu., Svitkin,Yu.V., Ugarova,T.Yu. and Agol,V.I. (1982) FEBS Lett. 141, 153-156.

19. Pelletier,J., Kaplan,G., Racaniello,V.R. and Sonenberg,N. (1988) J. Virol. 62, 2219-2227

20. Ugarova,T.Yu. (1987) Molek. Biol. (russ.) 21, 888-914.

21. Trono,D., Pelletier,J., Sonenberg,N. and Baltimore,D. (1988) Science 241, 445-448.

22. Trono,D., Andino,R. and Baltimore,D. (1988) J. Virol. 62, 2291-2299.

23. Del Angel,R.M., Papavassiliou,A.G., Fernandez-Tomas,C., Silverstein,S.J. and Racaniello,V.R. (1989) Proc. Natl. Acad. Sci. USA 86, 8299-8303.

24. Howell,M.T., Kaminski,A. and Jackson, R.J. (1989) in: 2nd International Symposium on Positive Strand RNA Viruses, abstracts, Vienna, Austria, p. 6. 